\title{
DVCS off the Neutron: E08-025 Jlab Hall A Experiment
}

\author{
Meriem BENALI*广 \\ LPC Caen, ENSICAEN, Université de Caen, CNRS/IN2P3, Caen, France \\ E-mail: benali@jlab.org
}

Malek MAZOUZ

Faculté des Sciences de Monastir, 5000 Monastir, Tunisia

\begin{abstract}
Generalized parton distributions (GPDs) are considered as suitable tools to study the internal structure of hadrons in terms of quarks and gluons. The Measurement of Deeply Virtual Compton Scattering (DVCS) cross sections is the simplest way to access GPDs. In particular, DVCS off the neutron is sensitive to GPD E, the least constrained GPD, which is needed to determine the quark angular momentum via Ji's sum rule. E08-025 experiment was performed in Jlab Hall A to measure the unpolarized cross section of photon electroproduction off a deuterium target at $Q^{2}=1.75 \mathrm{GeV}^{2}$ and $x_{B}=0.36$. After a brief description of the experimental setup, we will show the main steps of the data analysis. Finally, we will present our preliminary results on the photon electroproduction off the neutron and coherent deuteron, indicating for the first time a non-zero DVCS signal.
\end{abstract}

23rd International Spin Physics Symposium - SPIN2018 -

10-14 September, 2018

Ferrara, Italy

\footnotetext{
* Speaker.

${ }^{\dagger}$ For the Jefferson Lab Hall A Collaboration.
} 


\section{Introduction}

Lepton (electron or muon) scattering experiments have proven to be a powerful tool to investigate the dynamics of partons (quarks and gluons) inside the nucleon, governed by the Quantum Chromodynamics (QCD) theory. On the one hand, elastic scattering allows to probe the transverse charge and current distributions via the elastic form factors [1]. On the other hand, parton distribution functions (PDFs) measured in deep inelastic scattering (DIS) provide information about parton's longitudinal momentum and helicity distributions in the nucleon [2]. However, these two independent information are not correlated and do not allow a multi-dimensional description of the nucleon. Within the generalized parton distributions (GPDs) formalism, developed two decades ago, this multi-dimensional description has become possible. Indeed, GPDs are universal nonperturbative objects which encode correlations between the transverse position and the longitudinal momenta of partons [3]. In addition, GPDs give access to the contribution of quark angular momenta $J_{q}$ via Ji's sum rule and may contribute then to solve the nucleon spin puzzle [4]:

$$
J_{q}=\frac{1}{2} \int_{-1}^{+1} x d x\left[H_{q}(x, \xi, t=0)+E_{q}(x, \xi, t=0)\right] .
$$

At the leading order in $1 / Q$, the nucleon is described by eight GPDs for each quark flavor $q$. Four of them $\left(H_{q}, E_{q}, \widetilde{H}_{q}\right.$ and $\left.\widetilde{E}_{q}\right)$ conserve the helicity of the parton and the four others flip the helicity. Experimentally, GPDs can be accessed through hard exclusive processes such as Deeply Virtual Compton Scattering (DVCS) and Deeply Virtual Meson Production (DVMP). At the Bjorken limit $\left(Q^{2} \rightarrow \infty\right.$, energy of the virtual photon $v \rightarrow \infty$ and fixed Bjorken variable $\left.x_{B}=\frac{Q^{2}}{2 M v}\right)$, the DVCS amplitude can be factorized into a hard perturbative part $\left(\gamma^{*} q \rightarrow \gamma q\right)$ and a soft non-perturbative part parametrized by GPDs as shown in figure $1[5,6]$. This factorization theorem has been proven experimentally for $Q^{2}$ values as low as $2 \mathrm{GeV}^{2}[7]$.

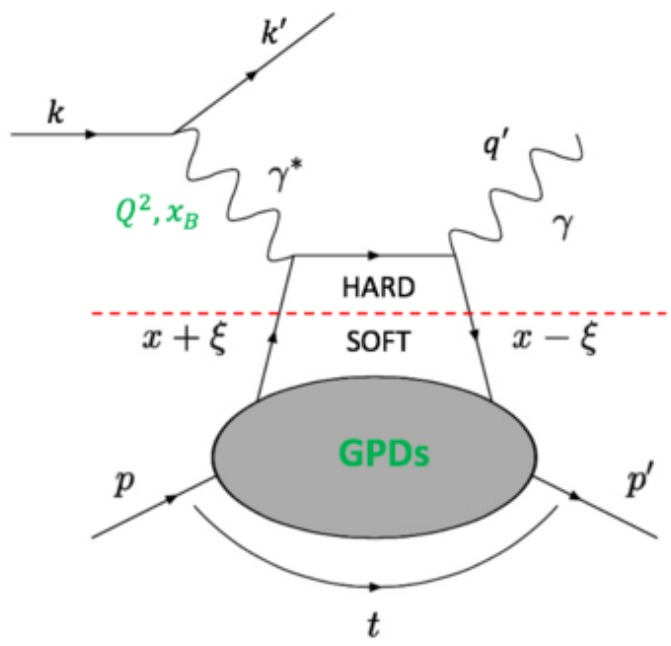

Figure 1: Leading order DVCS diagram. The hard perturbative calculable part is above the dashed line, where a virtual photon interacts with a single quark from the nucleon and a real photon is emitted. The non perturbative part (below the dashed line), encoded by GPDs, corresponds to the emission and absorption of a quark by the nucleon. 
Experimentally, the DVCS process interferes with the Bethe-Heitler $(\mathrm{BH})$ process where the real photon is emitted by the incoming or the scattered electron. We measure experimentally the photon electroproduction cross section which writes:

$$
\sigma(e N \rightarrow e N \gamma)=\left|\mathscr{T}_{B H}\right|^{2}+\left|\mathscr{T}_{D V C S}\right|^{2}+\mathscr{I}
$$

where $\mathscr{I}$ is the interference term. The BH amplitude $\left(\mathscr{T}_{B H}\right)$ is fully calculable using the nucleon form factors so measurements of the photon electroproduction cross section lead to the determination of the $\left|\mathscr{T}_{D V C S}\right|^{2}$ and the interference term. The beam energy dependence of the cross section allows to perform a separation between the latter terms, the so-called Rosenbluth separation.

Several DVCS experiments were performed in different facilities around the world [7-15], with different polarization configurations for the lepton beam and the target. These experiments were carried out in different kinematic domains in $\left(Q^{2}, x_{B}\right)$ to get several constraints on GPDs. In this proceeding, we discuss the E08-025 experiment which was performed in the Hall A of the Jefferson Lab in order to study the DVCS process off the neutron (n-DVCS) and extract its unpolarized cross section in the valence region $\left(x_{B}=0.36\right)$ at $Q^{2}=1.75 \mathrm{GeV}^{2}$. Contrary to the DVCS off the proton, The n-DVCS process is sensitive to the GPD E, the less constrained GPD, whose knowledge is needed to determine the total angular momentum of quarks (Eq. 1.1). The first experience dedicated to the study of the n-DVCS process is the E03-106 experiment performed in Hall A in 2004 [8]. Only the polarized cross section difference was measured in this pioneer experiment at a very close kinematic to the E08-025 one. Since no free neutron targets exist, we used a liquid deuterium (LD2) as a quasi-free neutron and proton target. The neutron contribution was then deduced by subtracting the data taken with a liquid hydrogen (LH2) target (E07-007 experiment [9]) from LD2 data.

\section{Experimental setup}

The data were taken in Jefferson Lab Hall A in 2010. A longitudinally polarized electron beam was incident on a LD2 target (15 cm long and $178 \mu \mathrm{m}$ thick). Both E07-007 and E08025 experiments ran concurrently with LH2 and LD2 targets being switched daily to minimize systematic uncertainties. Two beam energies $k_{0}=4.45 \mathrm{GeV}$ and $k_{0}=5.54 \mathrm{GeV}$ were used to perform the Rosenbluth separation between the DVCS ${ }^{2}$ and the interference terms. The beam polarization varied between $72 \%$ and $77 \%$, depending on the beam energy, and the beam current was about $3 \mu \mathrm{A}$ resulting on a luminosity around $10^{38} \mathrm{~cm}^{-2} \mathrm{~s}^{-1}$. As shown in figure 2 , the scattered electrons were detected in a High Resolution Spectrometer (HRS) which determines accurately the lepton kinematics. The emitted real photon was detected in an electromagnetic calorimeter composed of $13 \times 16 \mathrm{PbF}_{2}$ blocks, placed at $110 \mathrm{~cm}$ from the target, and centered around the virtual photon direction. This configuration leads to a momentum transfer $\mathrm{t} \in[-0.5,-0.1] \mathrm{GeV}^{2}$ and to a $[0,2 \pi]$ coverage in $\phi$ which is the angle between the hadronic and leptonic plans. The recoil nucleon, not detected in our experiment, is identified with the missing mass squared $M_{X}^{2}=$ $\left(q+p-q^{\prime}\right)^{2}$ where $q, p$ and $q^{\prime}=k-k^{\prime}$ are respectively the 4 -vectors of the real photon, the target nucleon and the virtual photon. 


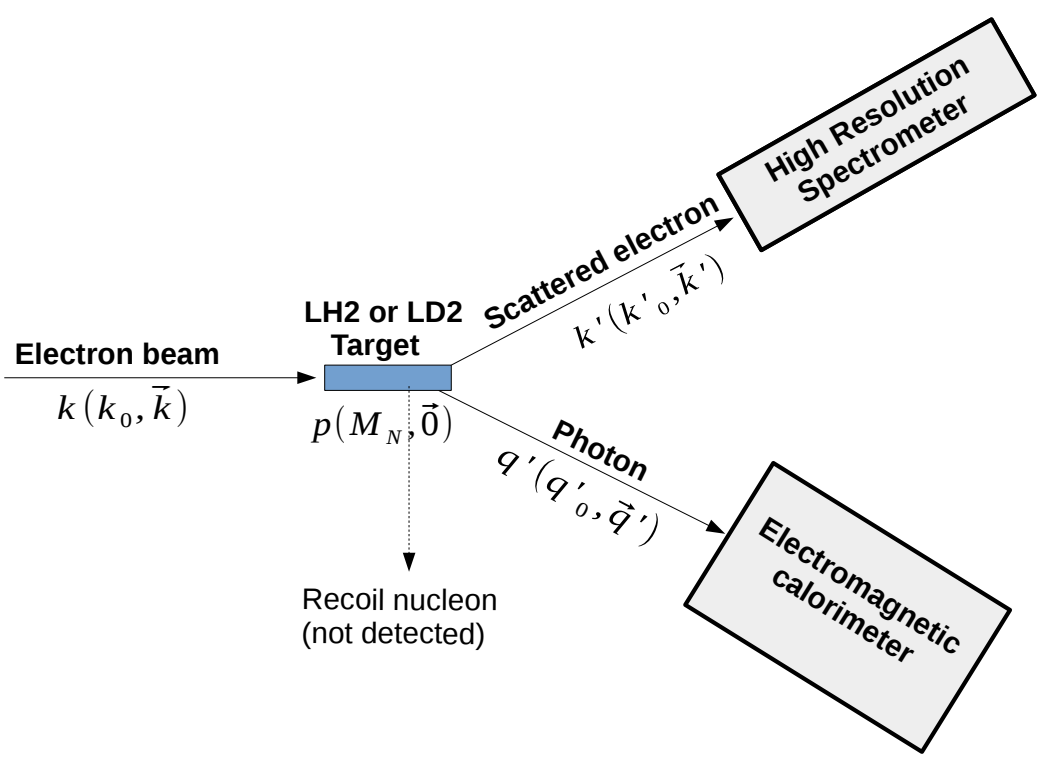

Figure 2: Experimental configuration of E07-007 and E08-025 experiments.

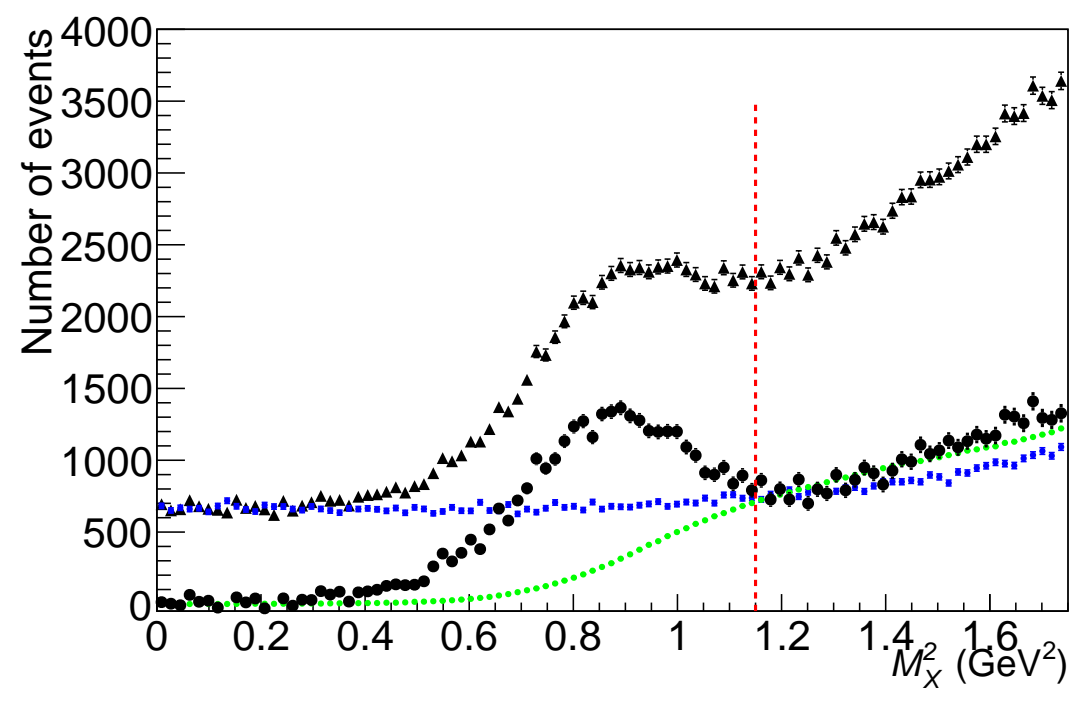

Figure 3: Missing mass squared $\left(M_{X}^{2}\right)$ distribution of LD2 data for $k_{0}=4.45 \mathrm{GeV}, Q^{2}=1.75 \mathrm{GeV}^{2}$ and $\langle t\rangle=-0.32 \mathrm{GeV}^{2}$. The raw data are represented by the blacks triangles. The contributions of accidentals and $\pi^{0}$ contamination events are shown respectively by the blue and the green spectrum. The remaining distribution after the subtraction of accidentals and $\pi^{0}$ contamination events is shown by the solid circles. The vertical dotted red line illustrates the pion production threshold.

\section{Data Analysis}

Two independent reactions are used to calibrate and monitor continuously the electromagnetic 
calorimeter : $H\left(e, e_{\text {Calo }}^{\prime} p_{H R S}\right)$ and $H, D\left(e, e_{H R S}^{\prime} \pi_{\text {Calo }}^{0}\right) X$ [16]. An energy resolution of $2.4 \%$ was achieved at $4.2 \mathrm{GeV}$ with a $\sim 3 \mathrm{~mm}$ spatial resolution. Figure 3 shows the $D(e, e \gamma) X$ missing mass squared distribution calculated with a target nucleon at rest. The exclusive photo production events are located in this spectrum around the nucleon squared mass $\left(M_{N}^{2} \approx 0.88 \mathrm{GeV}^{2}\right)$. Events below the pion production threshold $\left(M_{X}^{2}<\left(M_{N}+m_{\pi}\right)^{2} \approx 1.15 \mathrm{GeV}^{2}\right)$ correspond to $D(e, e \gamma) p n$ events and are contaminated by:

- Accidental events where the real photon detected in the calorimeter is in fortuitous coincidence with the scattered electron. The contribution of these events is estimated by analyzing events outside the coincidence window. The cut $M_{X}^{2}>0.5 \mathrm{GeV}^{2}$ is applied later to minimize this contribution.

- $\pi^{0}$ contamination events where the detected real photon comes from a $\pi^{0}$ asymmetric decay. The contribution of these events is determined by simulating all possible $\pi^{0} \rightarrow \gamma \gamma$ decays from exclusive $\pi^{0}$ electroproduction data [17, 18].

- Semi-inclusive events where at least a pion is produced in the final state in addition to the photon. These events are in principle above the $M_{X}^{2}$ pion production threshold but may contaminate the exclusive yield due to resolution effects. The cut $M_{X}^{2}<0.95 \mathrm{GeV}^{2}$ is applied later to minimize this contamination.

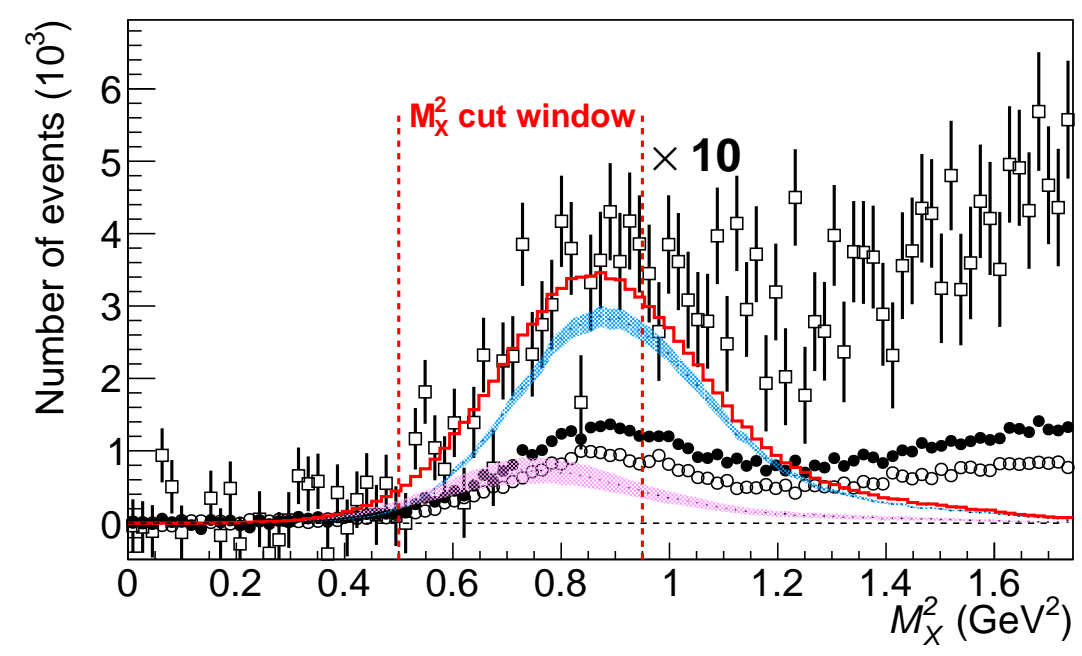

Figure 4: Missing mass squared distribution for the background free $D(e, e \gamma) X$ (solid circles) and normalized $H(e, e \gamma) X$ (open circles) data for $k_{0}=4.45 \mathrm{GeV}, Q^{2}=1.75 \mathrm{GeV}^{2}$ and $\langle t\rangle=-0.32 \mathrm{GeV}^{2}$. The difference between these spectra is shown by the open squares and is scaled by a factor 10 for clarity. The simulated $n(e, e \gamma) n$ and $d(e, e \gamma) d$ contributions are represented respectively by the blue and magenta bands (scaled by a factor 10) and are deduced from a global fit to the data (red histogram) in the exclusive $M_{X}^{2}$ region. The width of the bands represents the statistical uncertainty of the fit.

Figure 4 shows the $H(e, e \gamma) X$ and $D(e, e \gamma) X$ missing mass squared spectra after the subtraction of accidentals and the $\pi^{0}$ contamination events [19]. The Fermi momentum of bound protons inside 
the deuteron is added statistically to the $H(e, e \gamma) X$ data which are normalized to the same integrated luminosity of the $D(e, e \gamma) X$ data. The difference between $D(e, e \gamma) X$ and $H(e, e \gamma) X M_{X}^{2}$ distribution is also shown in figure 4. Events within the exclusivity cut $0.5<M_{X}^{2}<0.95 \mathrm{GeV}^{2}$ in the latter distribution correspond to :

$$
D\left(e, e^{\prime} \gamma\right) X-H\left(e, e^{\prime} \gamma\right) X=d\left(e, e^{\prime} \gamma\right) d+n\left(e, e^{\prime} \gamma\right) n
$$

where $d\left(e, e^{\prime} \gamma\right) d$ is the coherent-deuteron contribution which is shifted kinematically by $t / 2$ from the incoherent neutron contribution in the $M_{X}^{2}$ distribution. These data are divided into $12 \times 2 \times$ $5 \times 30$ bins in $\phi, k_{0}, t$ and $M_{X}^{2}$. The binning on the beam energy allows to perform the Resenbluth separation while the binning on $M_{X}^{2}$ allows to separate the $n\left(e, e^{\prime} \gamma\right) n$ and $d\left(e, e^{\prime} \gamma\right) d$ contributions.

\section{Extraction of the cross sections}

A Geant 4 based simulation is used to extract the photon electroproduction cross sections. This simulation takes into account the detector acceptances and the radiative effects. The calorimeter energy in the proton simulation $p\left(e, e^{\prime} \gamma\right) p$ is smeared to have a good matching between the experimental $H(e, e \gamma) p$ and the simulated data regarding the energy calibration and resolution. The obtained smearing coefficients are then applied to $n\left(e, e^{\prime} \gamma\right) n$ and $d\left(e, e^{\prime} \gamma\right) d$ simulated data. Finally the fermi-momentum is added statistically to the neutron simulated data.

Following Eq. (4.1), the unpolarized photon electroproduction cross section of the exclusive $\left(D\left(e, e^{\prime} \gamma\right) X-H\left(e, e^{\prime} \gamma\right) X\right)$ experimental data can be written as [20]:

$$
\frac{d^{4} \sigma}{d Q^{2} d x_{B} d t d \phi}=\sigma\left(B H_{n}\right)+\sigma\left(B H_{d}\right)+\sum_{i_{n}}\left(\Gamma_{i_{n}}\left(k_{0}, t, \phi\right) X_{i_{n}}(t)\right)+\sum_{i_{d}}\left(\Gamma_{i_{d}}\left(k_{0}, t, \phi\right) X_{i_{d}}(t)\right),
$$

where the $i_{n}\left(i_{d}\right)$ index refers to the neutron (coherent-deuteron) contribution, $\sigma(B H)$ is the known Bethe-Heitler cross section, $\Gamma_{i_{n / d}}\left(k_{0}, t, \phi\right)$ are calculable kinematical factors and $X_{i_{n / d}}$ are linear combinations of Compton Form Factors (CFFs) representing GPD integrals over $x$. The crosssection extraction is based on a global $\chi^{2}$ minimization between the experimental $N^{\exp }$ and the simulated $N^{\text {sim }}$ yields weighted by Eq. (4.1) cross section :

$$
\chi^{2}=\sum_{k=1}^{3600}\left(\frac{N_{k}^{\exp }-N_{k}^{\text {sim }}}{\delta_{k}^{\exp }}\right)^{2}
$$

where $\delta_{k}^{\text {exp }}$ is the statistical error on $N_{k}^{\exp }$ and $X_{i_{n / d}}(t)$ are the free parameters of the fit. Figure 5 shows an example of the total number of exclusive events as function of $\phi$. The distribution is symmetric relatively to $\phi=180$ degrees and is maximal around $\phi=0$. The simulated contributions of the neutron and coherent-deuteron $\mathrm{BH}$, determined within $1 \%$ uncertainty with the neutron and deuteron form factors, are also shown in figure 5 and exibit the same behavior as the experimental data. The difference between the total number of exclusive events and the sum of the neutron and coherent-deuteron BH indicates a non-zero DVCS signal.

The neutron and coherent-deuteron individual cross sections could be deduced after Eq. (4.2) minimization by injecting the $X_{i_{n / d}}(t)$ values in Eq. (4.1). The results, submitted for publication, 
show a significant deviation of the $n\left(e, e^{\prime} \gamma\right) n$ unpolarized cross sections from the $\mathrm{BH}$ at high $t$ values and represent the first observation of a positive n-DVCS signal. In addition, the combination of the neutron and proton results allows to determine the contribution of quarks $u$ and $d$ to the photon electroproduction cross section off the nucleon.

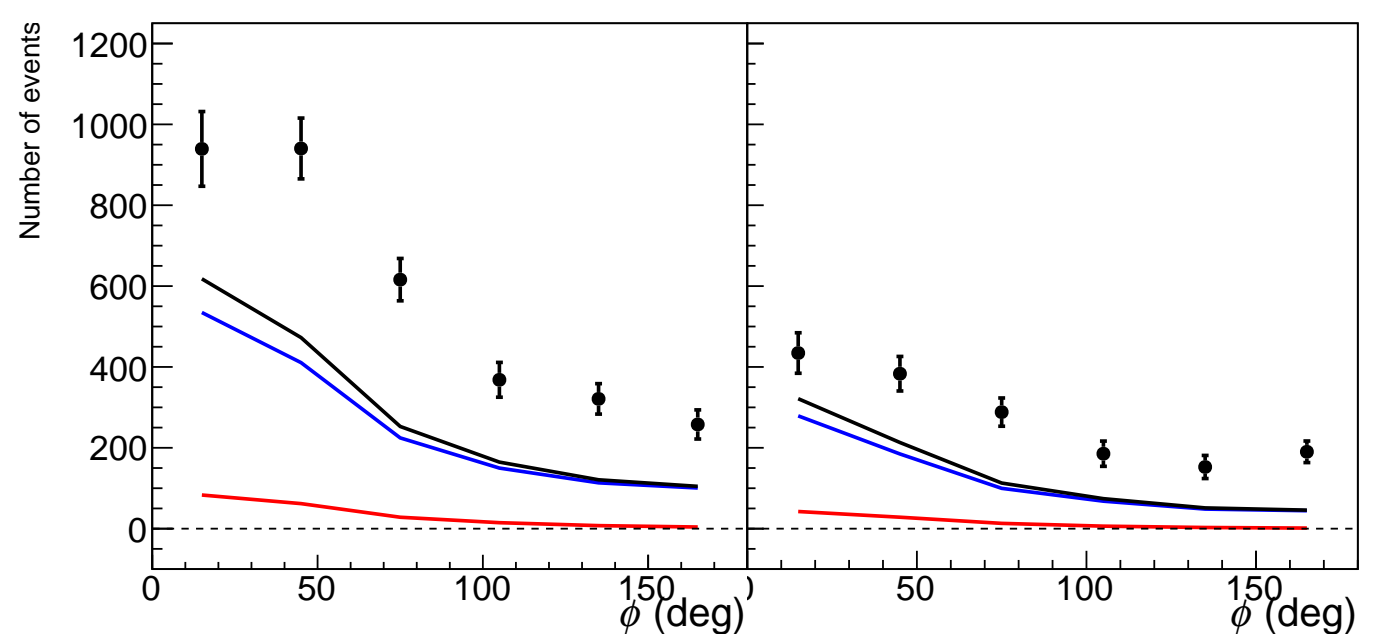

Figure 5: Number of events of $D\left(e, e^{\prime} \gamma\right) X-H\left(e, e^{\prime} \gamma\right) X$, with $0.5<M_{X}^{2}<0.95 \mathrm{GeV}^{2}$, as a function of $\phi$ for $E=4.45 \mathrm{GeV}^{2}$ (left) and $E=5.54 \mathrm{GeV}^{2}$ (right) for $\langle t\rangle=-0.32 \mathrm{GeV}^{2}$. The blue (red) histogram illustrates the neutron (coherent-deuteron) $\mathrm{BH}$ contribution. The sum of the two $\mathrm{BH}$ contributions is represented by the black histogram.

\section{Conclusion}

We have studied in E08-025 experiment the photon electroproduction process off the neutron in the valence region $\left(x_{B}=0.36\right)$ at $Q^{2}=1.75 \mathrm{GeV}^{2}$. The amplitude of this process is the sum of the known BH and the DVCS amplitude which encodes information about GPDs. In particular, DVCS off the neutron is sensitive to the E GPD needed in Ji's sum rule for the determination of the quark angular momenta. Our results show a significant deviation between the total number of exclusive photon electroproduction events and the corresponding BH contribution indicating for the first time, a positive DVCS signal. The unpolarized cross sections are then extracted for the neutron and coherent deutron. The study of the neutron is highly complementary to the proton since a flavor decomposition of the cross sections can be performed by combining proton and neutron results.

\section{References}

[1] V. Punjabi et al., The Structure of the Nucleon: Elastic Electromagnetic Form Factors, Eur. Phys. J. A51, 79 (2015) , [1503.01452] and references therein.

[2] H. Abramowicz et al, Combination of measurements of inclusive deep inelastic $e^{ \pm} p$ scattering cross sections and QCD analysis of HERA data, Eur. Phys. J. C75 (2015) 12, 580, [1506.06042] and references therein.

[3] M. Diehl, Generalized parton distributions, Phys. Rept. 388 (2003) 41, [hep-ph/0307382]. 
[4] X. Ji, Gauge-Invariant Decomposition of Nucleon Spin, Phys. Rev. Lett. 78, 610 (1997), [hep-ph/9603249].

[5] Collins, J.C. and Freund, A., Proof of factorization for deeply virtual Compton scattering in QCD, Phys. Rev. D59, 074009 (1999), [hep-ph/9801262].

[6] Ji, X. and Osborne, J., One-loop corrections and all order factorization in deeply virtual Compton scattering, Phys. Rev. D58, 094018 (1998), [hep-ph/9801260].

[7] C. Munoz Camacho et al., Scaling Tests of the Cross Section for Deeply Virtual Compton Scattering, Phys. Rev. Lett. 97, 262002 (2006), [nucl-ex/0607029].

[8] M. Mazouz et al., Deeply virtual compton scattering off the neutron, Phys. Rev. Lett. 99, 242501 (2007), [0709.0450].

[9] M. Defurne et al., A Glimpse of Gluons through Deeply Virtual Compton Scattering on the Proton, Nature Comm. 8 (2017) 1408, [1703.09442].

[10] M. Defurne et al., E00-110 experiment at Jefferson Lab Hall A : Deeply virtual Compton scattering off the proton at $6 \mathrm{GeV}$, Phys. Rev. C92, 055202 (2015), [1504.05453].

[11] A. Aktas et al., Measurement of deeply virtual Compton scattering at HERA, Eur. Phys. J. C44, 1 (2005), [hep-ex/0505061].

[12] C. Adlo et al., Measurement of deeply virtual Compton scattering at HERA, Phys. Lett. B517, 47 (2001), [hep-ex/0107005].

[13] S. Chekanov et al., Measurement of deeply virtual Compton scattering at HERA, Phys. Lett. B573, 46 (2003), [hep-ex/0305028].

[14] A. Airapetian et al., Measurement of Azimuthal Asymmetries With Respect To Both Beam Charge and Transverse Target Polarization in Exclusive Electroproduction of Real Photons, JHEP 2008-06, 066 (2008), [0802.2499].

[15] H. S. Jo et al., Cross sections for the exclusive photon electroproduction on the proton and Generalized Parton Distributions, Phys. Rev. Lett. 115, 212003 (2015), [1504.02009].

[16] M. Mazouz, Energy calibration of laterally segmented electromagnetic calorimeters based on neutral pion detection, Nucl. Sci. Tech. 28 (2017) 155.

[17] M. Mazouz et al., Rosenbluth separation of the $\pi^{0}$ electroproduction cross section off the neutron, Phys. Rev. Lett. 118, 222002 (2017), [1702.00835].

[18] M. Defurne, M. Mazouz et al., Rosenbluth separation of the $\pi^{0}$ electroproduction cross section, Phys. Rev. Lett. 117, 262001 (2016), [1608.01003].

[19] M. Benali, M. Mazouz and H. Fonvieille, Study of Photon Electroproduction on the Nucleon at High and Low Energy by Virtual Compton Scattering, Phys. Part. Nucl. 48, 1 (2017).

[20] A. Belitsky and D. Mueller, Exclusive electroproduction revisited: Treating kinematical effects, Phys. Rev. D82, 074010 (2010), [1005.5209]. 